\title{
On the Exact Constant in the Quantitative Steinitz Theorem in the Plane*
}

\author{
I. Bárány ${ }^{1}$ and A. Heppes ${ }^{2}$ \\ ${ }^{1}$ Mathematical Institute of the Hungarian Academy of Sciences, \\ P.O. Box 127, 1364 Budapest, Hungary \\ H2923bar@ella.hu \\ ${ }^{2}$ Hungaria Computing Ltd., Dózsa György u. 150, \\ 1134 Budapest, Hungary \\ H9202hep@ella.hu
}

\begin{abstract}
We determine the maximal value of $r$ with the following property. If the convex hull of a set in $R^{2}$ contains a unit circle $B$, then a subset of at most four points can be selected so that the convex hull of this subset contains the circle of radius $r$ concentric with $B$. That the result is sharp is shown by the example when the original set is the set of vertices of a regular pentagon circumscribed around $B$.
\end{abstract}

\section{Introduction}

Steinitz proved [S] a long time ago that if the interior of the convex hull of a set $X \subset R^{d}$ contains the point $p$, then there is a subset $Y \subset X$, of cardinality at most $2 d$, such that $p \in$ int conv $Y$. This result is made "quantitative" in [BKP] in the sense that, for every $d \geq 2$, there is a constant $c(d)$ with following property. If conv $X$ contains a closed ball of radius $r$ with center $p$, then $Y \subset X,|Y| \leq 2 d$, exists such that conv $Y$ contains the ball of radius $c(d) r$ with center $p$. It is shown in [BKP] that, in fact, $c(d)>d^{-2 d}$. The aim of this paper is to find the best constant $c(d)$ when $d=2$.

It turns out that, as expected, the worst case is when conv $X$ is a regular pentagon, $p$ is its center, and the ball is the inscribed circle of conv $X$.

\footnotetext{
* Imre Bárány was partially supported by Hungarian National Science Foundation Grant Nos. 1907 and 1909. Aladár Heppes was partially supported by Hungarian National Science Foundation Grant No. 2583.
} 
To simplify the presentation we assume that $X \subset R^{2}$ is finite, then $P=\operatorname{conv} X$ is a convex polygon. Let $B$ denote the unit circle centered at the origin and assume $B \subset P$. $r B$ is the circle of radius $r$ concentric with $B$. Define

$$
r(X)=\max _{Y \subset X,|Y| \leq 4} \max \{\rho: \rho B \subset \operatorname{conv} Y\},
$$

or, as in [KMY], define, more generally,

$$
r_{k}(X)=\max _{Y \subset X,|Y| \leq k} \max \{\rho: \rho B \subset \operatorname{conv} Y\} .
$$

When $X$ is the set of the vertices of a regular $(k+1)$-gon with inscribed ball $B$ we get

$$
r_{k}(X)=r_{k}^{*}=\frac{\cos (2 \pi /(k+1))}{\cos (\pi /(k+1))}=\left(1-\frac{3 \pi^{2}}{2(k+1)^{2}}\right)(1+o(1))
$$

as $k \rightarrow \infty$.

Theorem 1. Under the above hypothesis

$$
r(X)=r_{4}(X) \geq r_{4}^{*}=\frac{\cos 2 \pi / 5}{\cos \pi / 5}=\frac{3-\sqrt{5}}{2}=0.381966 \ldots
$$

Equality holds if and only if conv $X$ is a regular pentagon with inscribed circle $B$.

At the end of this paper we indicate how to prove the same result without assuming that $X$ is finite.

It is proved in [KMY] that, for all $X \subset R^{2}$ with $B \subset \operatorname{conv} X$,

$$
r_{k}(X) \geq 1-\frac{2 \pi^{2}}{k^{2}}
$$

It seems likely that, for every $k \geq 4, r_{k}(X) \geq r_{k}^{*}$ with equality only when $P$ is the regular $(k+1)$-gon. Theorem 1 proves this for $k=4$, i.e., $r_{4}(X) \geq r_{4}^{*}$ and, using the methods of this paper, we can extend this to $k=5$ :

Theorem 2. If $X \subset R^{2}$ is finite and $B \subset \operatorname{conv} X$, then

$$
r_{5}(X) \geq r_{5}^{*}=\frac{\cos 2 \pi / 6}{\cos \pi / 6}=\frac{1}{\sqrt{3}} .
$$

Equality holds if and only if conv $X$ is a regular hexagon with inscribed circle $B$. 
We can further show the validity of the inequality $r_{k}(X) \geq r_{k}^{*}$ for the case when $|X|=k+1$ :

Theorem 3. If $X \subset R^{2}$ is a set of $k+1$ points and $B \subset \operatorname{conv} X$, then

$$
r_{k}(X) \geq r_{k}^{*}
$$

\section{Proof of Theorem 3}

Let $X=\left\{a_{1}, \ldots, a_{k+1}\right\}$ (where $a_{k+i+1}=a_{i}$ ) be the set of vertices of a convex $(k+1)$-gon $P$ with $B \subset P$, the vertices indexed in cyclic order. Write $r=r_{k}^{*}$. We show that there is an $i$ such that

$$
r B \subset \text { int } \operatorname{conv}\left(X \backslash\left\{a_{i}\right\}\right)
$$

unless $P$ is a regular $(k+1)$-gon circumscribed around $B$.

If some diagonal of $P$ is disjoint from $r B$, the statement is trivially true, therefore we assume that, for all $i$,

$$
a_{i} a_{i+2} \cap r B \neq \varnothing
$$

Write $b_{i}$ and $c_{i}$ for the projection of the origin $o$ onto the line passing through $a_{i} a_{i+1}$ and $a_{i} a_{i+2}$, respectively. Set

$$
\begin{aligned}
\alpha_{i}=\angle a_{i} o b_{i}, & \beta_{i}=\angle b_{i} o a_{i+1}, \\
\varphi_{i}=\angle a_{i} o c_{i}, & \psi_{i}=\angle c_{i} o a_{i+2} .
\end{aligned}
$$

These angles are taken to be signed. First we assume that

$$
o \notin \text { int } \operatorname{conv}\left\{a_{i} a_{i+1} a_{i+2}\right\}, \quad i=1, \ldots, k+1,
$$

consequently, $\varphi_{i}, \psi_{i} \geq 0$. The conditions imply that

$$
\left|b_{i}\right|=\left|a_{i}\right| \cos \alpha_{i}=\left|a_{i+1}\right| \cos \beta_{i} \geq 1 \quad \text { and } \quad\left|c_{i}\right|=\left|a_{i}\right| \cos \varphi_{i}=\left|a_{i+2}\right| \cos \psi_{i} \leq r
$$

So we have

$$
\begin{gathered}
\cos \varphi_{i} \leq \frac{r}{\left|a_{i}\right|} \leq r \cos \alpha_{i} \quad \text { or } \quad \varphi_{i} \geq \arccos \left(r \cos \alpha_{i}\right) \\
\cos \psi_{i} \leq \frac{r}{\left|a_{i+2}\right|} \leq r \cos \beta_{i+1} \quad \text { or } \quad \psi_{i} \geq \arccos \left(r \cos \beta_{i+1}\right) .
\end{gathered}
$$


The second derivative of the function $f(t)=\operatorname{arc} \cos (r \cos t)$ is positive when $t \in(-\pi / 2, \pi / 2)$. So $f(t)$ is convex and the Jensen inequality implies that

$$
\begin{aligned}
4 \pi & =\sum_{1}^{k+1}\left(\varphi_{i}+\psi_{i}\right) \geq \sum_{1}^{k+1}\left(\arccos \left(r \cos x_{i}\right)+\arccos \left(r \cos \beta_{i+1}\right)\right) \\
& \geq 2(k+1) \arccos \left(r \cos \frac{2 \pi}{2(k+1)}\right) \\
& =2(k+1) \arccos \left(\cos \frac{2 \pi}{k+1}\right)=4 \pi .
\end{aligned}
$$

Thus equality holds in all the inequalities above. This proves the claim under assumption (2.1).

Suppose now that assumption (2.1) does not hold for some "exceptional" values of $i$. We show the existence of another $(k+1)$-gon $P^{\prime}$ containing $B$ that has fewer exceptional $i$ values. Further, $P^{\prime}$ has the property that if the convex hull of a $k$-subset of $X$ contains $\rho B$, then there are $k$ vertices of $P^{\prime}$ such that their convex hull contains $\rho B$.

Let $i=1$ be an exceptional value, i.e., $o \in$ int $\operatorname{conv}\left\{a_{1} a_{2}, a_{3}\right\}$. Let $L$ be the line, parallel to $a_{1} a_{3}$ and passing through the origin. It meets the lines $a_{k} a_{1}$ and $a_{5} a_{3}$ in points $a_{1}^{\prime}$ and $a_{3}^{\prime}$, respectively. Now replace $a_{1}$ by $a_{1}^{\prime}$ and $a_{3}$ by $a_{3}^{\prime}$ to get $P^{\prime}$. Clearly, $P \subset P^{\prime}$ and, except for $a_{1} \mathrm{a}_{3}$, all diagonal lines of type $a_{i} a_{i+2}$ remain the same. From this all the above claims follow, concluding the proof of Theorem 3.

\section{Proof of Theorem 1}

In the proof $r$ stands for $r_{4}^{*}$. We assume, without loss of generality, that $X$ coincides with the set of vertices of an $n$-gon $P$. We call the elements of $X$ vertices (of $P$ ) and denote them by $a, b, c, \ldots$. The diagonal $a b$ is called an inner (outer) diagonal if $a b \cap r B \neq \varnothing$ is true (false).

We assume that

$r B$ is not contained in int conv $Y$ for any $Y \subset X,|Y| \leq 4$.

We derive several properties of $X$ and finally conclude that $P$ has to be a regular pentagon circumscribed around $B$.

Claim 1. To every vertex a two distinct inner diagonals adjacent to a exist.

Proof. The $(n-3)$ diagonals starting at $a$ dissect $P$ into $(n-2)$ triangles. If the minimal number of those (consecutive) triangles which cover $r B$ is less than three, then we are in contradiction with (*). Else we have at least two inner diagonals. 
Definition. A sequence $a_{1}, a_{2}, \ldots, a_{k}$ of consecutive vertices is a critical sequence if $a_{i} a_{j}$ is an inner diagonal if and only if $\{i, j\}=\{1, k\}$.

For a critical sequence, $k \geq 3$ holds, since no edge of $P$ can intersect $r B$. Further, for any $i \in\{2, \ldots, k-1\}$, the diagonals $a_{1} a_{i}$ and $a_{i} a_{k}$ avoid $r B$. Now let $a_{k}$, $a_{k+1}, \ldots, a_{n}, a_{1}$ be the rest of the vertices listed consecutively from $a_{k}$ to $a_{1}$. This sequence cannot be critical since if it was, then by the previous remark, for any $i \in\{2, \ldots, k-1\}$ and $j \in\{k, \ldots, n\}$,

$$
r B \subset \text { int } \operatorname{conv}\left\{a_{1}, a_{i}, a_{k}, a_{j}\right\}
$$

contradicting (*). So we may and do speak of a critical diagonal and of its critical side since this sequence (side) is uniquely determined.

Remark 1. It is evident that on both sides of an inner diagonal $a b$ there is (at least) one critical diagonal (one of them, actually, may coincide with it). Claim 1 implies that at least two critical diagonals exist.

We need some further notation. If $x, y \in R^{2}(x \neq y)$, then we write $\overline{x y}$ for the line they span and $\overrightarrow{x y}$ for the half-line starting at $x$ and containing $y$. Let cone $(\overrightarrow{x y}, \overrightarrow{u v})$ denote the convex cone whose boundary is contained in $\overrightarrow{x y} \cup \overrightarrow{u v}$. (This makes sense only if the half-lines $\overrightarrow{x y}$ and $\overrightarrow{u v}$ intersect.) Define the angle $\gamma$ by $\sin \gamma=r, 0<\gamma<\pi / 2$. It is clear that, for any inner diagonal $a b$,

$$
\angle a o b \geq \pi-2 \gamma
$$

Here and in what follows $\angle a o b$ denotes the (unsigned) angle of the half-lines $\overrightarrow{o a}$ and $\overrightarrow{o b}$. As luck would have it,

$$
\gamma<\frac{\pi}{8}
$$

In fact $\gamma=0.391922 \ldots$ and $\pi / 8=0.392699 \ldots$, which can be verified by simple calculation. We refer quite often to this fact. We also need the following:

Lemma 1. Assume $a b$ is an outer diagonal or an edge of $P$. Let $\overrightarrow{a x}$ and $\overrightarrow{b y}$ be tangent half-lines to $r B$ such that the triangle determined by $\overline{a x}, \overline{b y}$, and $\overline{a b}$ contains $r B$. If the intersection of the lines $\overline{a x}, \overline{b y}$ is in int $B$, then there are vertices $u, v \in X$ such that

$$
r B \subset \text { int } \operatorname{conv}\{a, b, u, v\}
$$

Proof. The apex of the cone $C=\operatorname{cone}(\overrightarrow{a x}, \overrightarrow{b y})$ is in int $B$. Then $C$ intersects the boundary of $P$, and it intersects the interior of some edge $u v$ of $P$, and Lemma 1 follows immediately. 


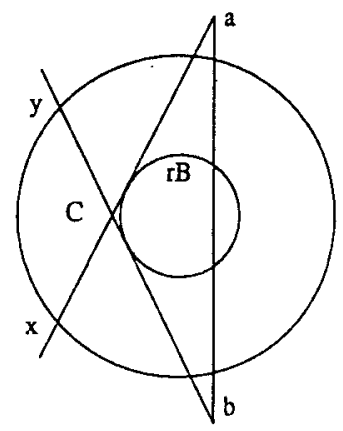

Fig. 1

Now let $a b$ be a critical diagonal. Draw a half-line starting from $a$ (resp. from b) tangent to $r B$ and toward the noncritical side of $a b$. Denote the last point of this half-line in $B$ by $x$ (resp. $y$ ) (see Fig. 1).

Claim 2. No vertex of $P$ lies in the interior of $C=\operatorname{cone}(\overrightarrow{a x}, \overrightarrow{b y})$.

Proof. Assume $u \in X \cap$ int $C$ and let $c$ be any vertex on the critical side of $a b$. Then $r B \subset \operatorname{conv}\{a, b, c, u\}$.

Claim 3. If $a b$ is a critical diagonal, then the origin lies on its noncritical side.

Proof. Assume the contrary and consider the previous picture. It is easily seen (as a consequence of the inequality $\gamma<\pi / 8$ ) that the apex of the cone $C=$ cone $(\overrightarrow{a x}, \overrightarrow{b y})$ is in int $B$. As $C$ intersects the boundary of $P$, and, by Claim 2 , there is no vertex in int $C$, an edge $u v$ crossing $C$ (and not intersecting $B$ ) exists. We choose the notation so that $a u$ is a diagonal meeting $r B$. Let $\overrightarrow{u u^{\prime}}$ and $\overrightarrow{v v^{\prime}}$ be half-lines, tangent to $r B$ such that the triangle determined by $\overline{u v}, \overline{u u^{\prime}}$, and $\overline{v v^{\prime}}$ contains $r B$. We choose $u^{\prime}$ and $v^{\prime}$ as the last point in $B$, respectively (see Fig. 2).

We show now that the lines $\overline{u u^{\prime}}$ and $\overline{v v^{\prime}}$ meet in int $B$. In view of Lemma 1 this will prove Claim 3. We compute angles: $\angle a o u \geq \pi-2 \gamma, \angle u o u^{\prime} \geq \pi-2 \gamma$, therefore $\angle a o u^{\prime} \leq 4 \gamma$. Similarly, $\angle b o v^{\prime} \leq 4 \gamma$. Since $o$ is on the same side of $a b$ as $u^{\prime}$ and $v^{\prime}$, we get

$$
\angle a o u^{\prime}+\angle b o v^{\prime}+\angle a o b \leq \pi+8 \gamma<2 \pi
$$

consequently the lines $\overline{u u^{\prime}}$ and $\overline{v v^{\prime}}$ intersect in int $B$.

Claim 4. There is at most one critical diagonal on each side of any line L passing through the origin. 


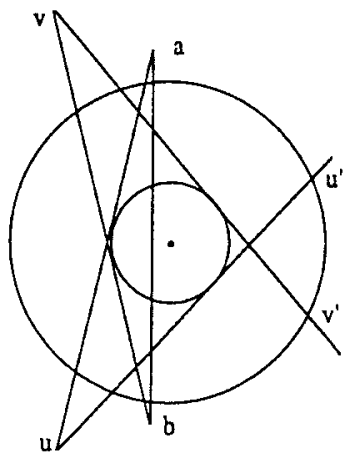

Fig. 2

Proof. Let $p$ and $q$ be the points where $L$ crosses the boundary of $P$ and assume there are two critical diagonals $a_{1} b_{1}$ and $a_{2} b_{2}$ on one side of $p q$ (Fig. 3). The order of the points in question along the boundary of $P$ is $p, a_{1}, a_{2}, b_{1}, b_{2}, q\left(p=a_{1}\right.$ and $q=b_{2}$ are allowed). By Claim 3, each of $a_{1} b_{1}$ and $a_{2} b_{2}$ is critical with respect to the side not containing the origin. Then $b_{1} a_{2} \cap r B=\varnothing$ and we can apply Lemma 1 if the corresponding tangents $\overline{a_{2} x}$ and $\overline{b_{1} y}$ meet in int $B$. (x and $y$ are chosen to be again on the boundary of $B$ so that both cone $\left(\overrightarrow{b_{1} y}, \overrightarrow{b_{1} a_{2}}\right)$ and cone $\left(\overrightarrow{a_{2} x}, \overrightarrow{a_{2} b_{1}}\right)$ contain $r B$.) We compute angles:

$$
\angle q o x=2 \pi-\left(\angle q o b_{2}+\angle b_{2} o a_{2}+\angle a_{2} o x\right) \leq 4 \gamma
$$

and

$$
\angle p o y=2 \pi-\left(\angle p o a_{1}+\angle a_{1} o b_{1}+\angle b_{1} o y\right) \leq 4 \gamma
$$

and so

$$
\angle q o x+\angle p o y \leq 8 \gamma<\pi
$$

consequently, the lines $\overline{a_{2} x}$ and $\overline{b_{1} y}$ meet in int $B$.

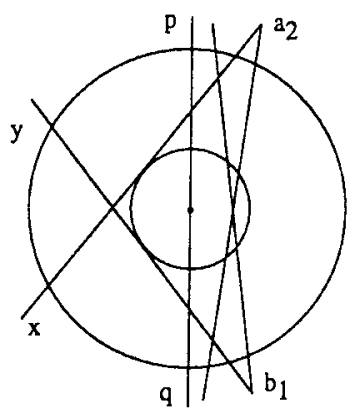

Fig. 3 
Claim 5. For any inner diagonal there is exactly one critical diagonal on the side not containing the origin. (The two diagonals may coincide.)

Proof. Immediate from Claims 3 and 4.

We say that two diagonals are disjoint if they have no inner point in common.

Claim 6. To every critical diagonal ab there are at most two critical diagonals disjoint from it.

Proof. If there were three such diagonals, then the three lines they span would intersect the half-lines $\overrightarrow{a b}$ and $\overrightarrow{b a}$ in two or three points (one can be parallel to $a b$ ). Then one of them, say $\overrightarrow{a b}$ is met by them in at most one point. We show that this is impossible.

Assume that the lines of two critical diagonals $c e$ and $d f$ do not meet $\overrightarrow{a b}$ (as in Fig. 4). Then $\operatorname{de} \cap r B=\varnothing$. Consider, again, the tangent half-lines $\overrightarrow{d x}$ and $\overrightarrow{e y}$ of $r B$, with $x$ and $y$ being the last points of the half-lines in $B$, and $r B$ lying in the triangle determined by the lines $\overline{d x}, \overline{e y}, \overline{d e}$. We show that these two half-lines meet inside $B$. Then Lemma 1 can be applied and Claim 6 follows. Let $a^{\prime} b^{\prime}$ and $a^{\prime \prime} b^{\prime \prime}$ be the two chords of $B$ tangent to $r B$ and parallel to $a b$ on the critical and noncritical side, respectively, $a^{\prime}$ and $a^{\prime \prime}$ being closer to $a$ than to $b$; further, let $c^{\prime}$, $d^{\prime}$, and $e^{\prime}$ be the last points of the half-lines $\overrightarrow{e c}, \overrightarrow{f d}$, and $\overrightarrow{c e}$ in $B$, respectively. Clearly, $c^{\prime} \in \operatorname{arc}\left(a^{\prime}, a^{\prime \prime}\right)$ and $d^{\prime} \in \operatorname{arc}\left(a^{\prime}, a^{\prime \prime}\right)$. This implies, on the one hand, $x \in \operatorname{arc}\left(s, b^{\prime}\right)$, and, on the other hand, $e^{\prime} \in \operatorname{arc}\left(t, b^{\prime}\right) \Rightarrow y \in \operatorname{arc}\left(t^{\prime}, a^{\prime}\right)$, where $s\left(\neq b^{\prime \prime}\right), t\left(\neq b^{\prime}\right)$, and $t^{\prime}\left(\neq a^{\prime}\right)$ denote the last points in $B$ of the half-lines $\overrightarrow{a^{\prime \prime} s}, \overrightarrow{a^{\prime} t}$, and $\overrightarrow{t t^{\prime}}$, all tangent to $r B$. From the relations

$$
\angle a^{\prime} o s=\pi-4 \gamma>\frac{\pi}{2} \quad \text { and } \quad \angle t^{\prime} o a^{\prime}=4 \gamma<\frac{\pi}{2}
$$

our claim follows directly.

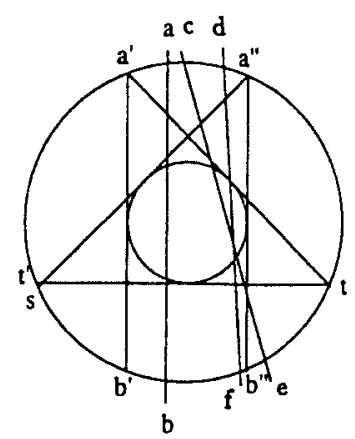

Fig. 4 


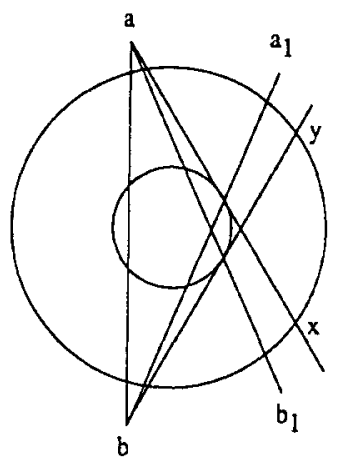

Fig. 5

Claim 7. To every critical diagonal ab there are exactly two critical diagonals disjoint from it.

Proof. By Claim 1 from each end of $a b$ a further inner diagonal starts (necessarily into the noncritical side.) Let $x$ and $y$ be the last point within $B$ of the half-lines starting from $a$ and $b$, tangent to $r B$ on the noncritical side of $a b$, respectively, further, let $b_{1}\left(a_{1}\right)$ be the "last" vertex for which $a b_{1}\left(b a_{1}\right)$ is an inner diagonal, i.e., cone $\left(\overrightarrow{b a_{1}}, \overrightarrow{b y}\right)$ and cone $\left(\overrightarrow{a b_{1}}, \overrightarrow{a x}\right)$ contain no vertex besides $a_{1}$ and $b_{1}$, respectively (Fig. 5). By Remark 1 to both $a b_{1}$ and $b a_{1}$ there is a critical diagonal on the non- $a b$ side. We show that they do not coincide. Since by Claim 2 int cone $(\overrightarrow{a x}, \overrightarrow{b y})$ is free from vertices, there is no vertex in int cone $\left(\overrightarrow{a b_{1}}, \overrightarrow{b a_{1}}\right)$. Thus $a_{1} b_{1}$ is an edge and by this the last possibility for a common critical diagonal is excluded.

Remark 2. The proof shows that if there are two critical diagonals ce and $d f$ disjoint from $a b$, then none of them is parallel to $a b, \overline{c e}$ intersects one of the half-lines $\overrightarrow{a b}$ and $\overrightarrow{b a}$, and $\overrightarrow{d f}$ intersects the other one.

We say that the two critical diagonals intersect if they are not disjoint.

Claim 8. There are no three critical diagonals that pairwise intersect.

Proof. Assume, to the contrary, that $a b, c d$, ef are pairwise intersecting critical diagonals, and let $a, c, e, b, d, f$ be the order of these vertices along the perimeter of $P$. We assume, without loss of generality, that $e$ is on the critical side of $a b$ and $b$ is on the critical side of $e f$. There are two cases to consider now:

Case 1: $a$ and $f$ are on the critical side of $c d$. Then, by Claim 3, the origin lies in the triangle determined by the lines $\overline{a b}, \overline{c d}$, $\overline{e f}$ and this triangle is contained in conv $\{a, e, d\}$ (Fig. 6). However, $a e, e d, d a$ avoid $r B$ so

$$
r B \subset \text { int } \operatorname{conv}\{a, e, d\},
$$




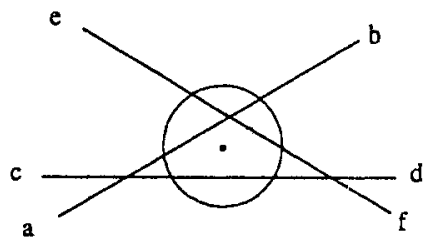

Fig. 6

Case 2: $e$ and $b$ are on the critical side of $c d$. Then $o \in \operatorname{conv}\{a, e, b, f\}$ since otherwise we separate conv $\{a, e, b, f\}$ from $o$ by a line $L$ passing through the origin and observe that two (in fact, three) critical diagonals lie on one side of $L$, contradicting Claim 4. If af $\cap r B=\varnothing$, then

$$
r B \subset \text { int } \operatorname{conv}\{a, e, b, f\}
$$

and we are finished. However, if $a f \cap r B \neq \varnothing$, then there is a critical diagonal on the nonzero side of af (by Claim 5), and there are three critical diagonals on the zero side of that diagonal, contradicting Claim 7.

Define now a graph $\Gamma$ whose vertex set consists of the critical diagonals, and two of them are joined by an edge if the two critical diagonals are disjoint.

Claim 9. $\Gamma$ is a five-cycle.

Proof. By Claim 7 every degree in $\Gamma$ is two, thus $\Gamma$ is the union of finitely many cycles.

A critical diagonal is seen from $o$ at an angle at least $\pi-2 \gamma$. Then three pairwise disjoint diagonals would give a total of at least $3(\pi-2 \gamma) \geq 2 \pi$, showing that there is no triangle $C_{3}$ among the cycles of $\Gamma$. A four-cycle $C_{4}$ in $\Gamma$ corresponds to critical diagonals $D_{1}, D_{2}, D_{3}, D_{4}$ where $D_{1}$ intersects $D_{2}$ and $D_{3}$ intersects $D_{4}$ and the rest of the pairs are disjoint. Set $D_{i}=a_{i} b_{i}$. Then $\operatorname{conv}\left\{a_{1}, b_{1}, a_{2}, b_{2}\right\}$ and $\operatorname{conv}\left\{a_{3}, b_{3}, a_{4}, b_{4}\right\}$ can be separated by a line $L$, and the nonzero side of $L$ would contain two critical diagonals which is not allowed by Claim 4 . So there is no $C_{4}$ in $\Gamma$. A $k$-cycle with $k \geq 6$ would contain an independent set of three which is excluded by Claim 8 . Thus $\Gamma$ consists of five-cycles only. However, two distinct five-cycles would again contain an independent triple. Consequently, $\Gamma$ is a single five-cycle.

We are homing in on the target now. Let $D_{i}, i=1, \ldots, 5\left(D_{i+5}=D_{i}\right)$, be the five critical diagonals, the consecutive pairs disjoint in cyclic order (Fig. 7). Set $D_{i}=a_{i} b_{i} . D_{2}$ is disjoint from $D_{1}$ and $D_{3}$ and both of them are on the noncritical side of $D_{2}$. In view of Remark 2 (following Claim 7) we can choose the notation so that $\overrightarrow{a_{1} b_{1}}$ intersects $\overrightarrow{b_{2} a_{2}}$, and $\overrightarrow{a_{3} b_{3}}$ intersects $\overrightarrow{a_{2} b_{2}} . D_{4}$ is disjoint from $D_{3}$ and intersects $D_{1}$ and $D_{2}$. Let $b_{4}$ (resp. $\left.a_{4}\right)$ be the critical side of $a_{2} b_{2}\left(a_{1} b_{1}\right)$. $D_{5}$ is 


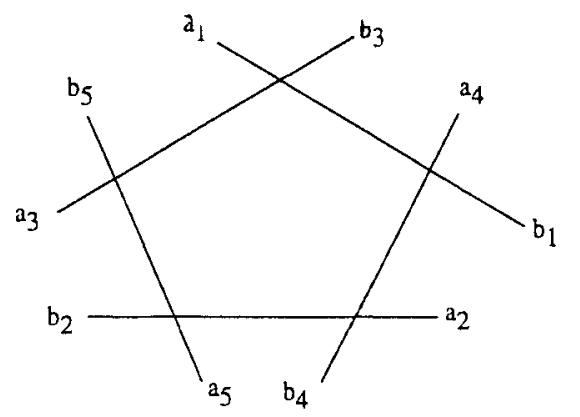

Fig. 7

disjoint from $D_{4}$ and $D_{1}$ and it intersects $a_{3} b_{3}$. Thus the order of these points on the boundary of $P$ is $a_{1}, b_{3}, a_{4}, b_{1}, a_{2}, b_{4}, a_{5}, b_{2}, a_{3}, b_{5}$.

It follows from Remark 2 that $\overrightarrow{a_{i} b_{i}}$ intersects $\overrightarrow{b_{i+1} a_{i+1}}$. Let $c_{i}$ denote the point of intersection. Notice that $c_{i}=b_{i}=a_{i+1}$ is possible. We show next that there is no vertex in the interior of the cone $\left(\overrightarrow{b_{i} a_{i}}, \overrightarrow{a_{i+2} b_{i+2}}\right)(i=1, \ldots, 5)$. Assume, to the contrary, that $v$ is a vertex in int cone $\left(\overrightarrow{b_{1} a_{1}}, \overrightarrow{a_{3} b_{3}}\right)$, say. Then all four edges of conv $\left\{v, b_{2}, a_{5}, a_{2}\right\}$ are disjoint from $r B$ and since $a_{2} b_{2}$ is an inner diagonal, $r B \subset$ int $\operatorname{conv}\left\{v, b_{2}, a_{5}, a_{2}\right\}$ contradicting (*). This means that all vertices are contained in the star-pentagon $c_{1} c_{2} c_{3} c_{4} c_{5}$. Set $Q=\operatorname{conv}\left\{c_{1}, \ldots, c_{5}\right\}$. Observe that

$$
Q \supset P \supset B
$$

and the first inclusion is strict unless $c_{i}=b_{i}=a_{i+1}$ for every $i=1, \ldots, 5$. Then, by Theorem 3, either $Q$ is a regular pentagon circumscribed around $B$ or it has a diagonal not meeting $r B$. Since every diagonal of $Q$ intersects $r B$, the first alternative holds. As $B$ is the incircle of $Q$ the inclusion $P \subset Q$ cannot be strict, consequently $P=Q$.

\section{Extensions}

Sketch of the Proof of Theorem 1 when $X$ is not Finite. Write $P=\operatorname{conv} X$, $\bar{X}=\operatorname{cl} X, \bar{P}=\mathrm{cl} \operatorname{conv} X=\mathrm{cl} P$. There are two cases to consider.

Case 1: $X$ is bounded. Then $P$ is bounded, too, and $\bar{X}, \bar{P}$ are compact. It can be readily seen that the proof of Theorem 1 goes through (almost without change) for the compact set $\bar{X}$. Then either $\bar{P}$ is a regular pentagon circumscribed around $B$ or there are points $y_{1}, y_{2}, y_{3}, y_{4} \in X$ such that $r B \subset$ int $\operatorname{conv}\left\{y_{1}, \ldots, y_{4}\right\}$. As $y_{i} \in \bar{X}$, there are points in $X$ arbitrarily close to $y_{i}$. So for suitable $x_{1}, x_{2}, x_{3}, x_{4} \in X$ we have $r B \subset$ int $\operatorname{conv}\left\{x_{1}, \ldots, x_{4}\right\}$.

When $\bar{P}$ is a regular pentagon, then so is $P$ as well but with some points on its boundary missing from $P$. A simple analysis using the fact that $P$ contains the closed disk $B$ finishes the proof. 
Case 2: $X$ is not bounded. We may assume that $B$ is not contained in the convex hull of any bounded subset of $X$, i.e., for all $R>0$,

$$
B \not \subset \operatorname{conv}(R B \cap X) \text {. }
$$

It can be shown, then, that as $R \rightarrow \infty$ the sets

$$
\operatorname{cl}(B \backslash \operatorname{conv}(R B \cap X))
$$

shrink to one or two points on the boundary of $B$. Let $b$ be this point (or one of these points). Clearly, $b \in X$ must hold. Further, one of the half-lines, $L$, starting from $b$ and tangent to $B$, has the property that $X$ contains points arbitrarily close to $L$ and arbitrarily far from $b$. Let $\overrightarrow{b x}$ be the half-line tangent to $r B$ (so that $r B \subset \operatorname{cone}(L, \overrightarrow{b x}))$, where $x$ is a point on the boundary of $B$. We show easily that $x \in \operatorname{conv}\{a, b, c\}$ for some $a, c \in X$. Then, for a suitable $y \in X$ far from $b$ but close to $L$, we get

$$
r B \subset \text { int } \operatorname{conv}\{a, b, c, y\} .
$$

Sketch of the Proof of Theorem 2. Let $X$ be finite and set $r=r_{5}^{*}$. The definition of a critical diagonal is the same as in Theorem 1 . Let $a b$ be a critical diagonal and let $a c$ (resp. $b d$ ) be the last inner diagonal adjacent to $a(b)$ and distinct from $a b$ (see Claim 1 from the proof of Theorem 1). The lines $\overline{a c}$ and $\overline{b d}$ intersect outside $B$ since otherwise $r B \subset$ int conv $\{a, b, c, d, x\}$ for any $x \in X$ from the critical side of $a b$. Then the nonzero side of $a c(b d)$ contains a critical diagonal $a_{1} c_{1}\left(b_{1} d_{1}\right)$. Thus the critical diagonals $D_{1}=a_{1} b_{1}, D_{2}=a_{1} c_{1}$, and $D_{3}=b_{1} d_{1}$ are pairwise disjoint. We can find three pairwise disjoint critical diagonals $E_{1}, E_{2}$, and $E_{3}$ so that $E_{i}$ is disjoint from $D_{j}$ if and only if $i=j$. (We omit the details.) These six diagonals form a star-hexagon. The rest of the proof is analogous to that of Theorem 1.

Some related problems are discussed in [RZ].

\section{Acknowledgment}

We thank two anonymous referees for careful reading and many valuable comments.

\section{References}

[BKP] I. Bárány, M. Katchalski, and J. Pach, Quantitative Helly-type theorems, Proc. Amer. Math. Soc., 86 (1982), 109-114.

[KMY] D. Kirkpatrick, B. Mishra, and C.-K. Yap, Quantitative Steinitz's theorem with applications to multifingered grasping, Discrete Comput. Geom. 7 (1992), 295-307.

[RZ] J. Reay and T. Zamfirescu, Interiors of uniform size in Steinitz's theorem, in Discrete Geometrie III (A. Florian, ed.), University of Salzburg, 1985, pp. 319-328.

[S] E. Steinitz, Bedingt konvergente Reihen und konvexe Systeme, J. Reine Angew. Math., 144 (1913), 128-175, 145 (1914), 1-40, 146 (1916), 1-52. 